\title{
Tissue Engineering: From Bench to Bed Side Exemples of Applications
}

\author{
J-F. Stoltz ${ }^{1,2}$, C. Huselstein ${ }^{1}$, D. Bensoussan ${ }^{1}$, N. de Isla ${ }^{1}$, V. Decot ${ }^{1}$ \\ ${ }^{1}$ CNRS UMR 7561, Biopôle, Faculté de Médecine - Université Lorraine, VANDOEUVRE LES NANCY, France \\ ${ }^{2}$ CHU de Nancy, UTCT, Brabois, VANDOEUVRE LES NANCY, France
}

Received 2012

\begin{abstract}
Tissue engineering is an emerging multidisciplinary field involving surgery medicine, biology, chemistry, mechanics and engineering to improve the health and quality of life by restoring, maintaining or enhancing tissue and organ functions [6;11]. The principle is simple: cells are collected and introduced -with or without modification of their biological properties- into an environment in which physico-chemical and mechanical parameters are kept stable. When they reach maturity, the biotissue or cells can be grafted. The main parameters are: cells, scaffold, biological molecules and mechanical forces. A combination of these parameters can promote cellular differentiation and proliferation. But it should be emphasized that at this time, signalling pathways and the rationale behind the physiological design remain to be elucidated. Among the main medium-term clinical applications are cardiac insufficiency, atherosclerosis, osteoarthritis, diabetes, liver diseases, bladder, and skin. In this paper the examples of cartilage and vascular engineering will be examined.
\end{abstract}

Keywords: Tissue Engineering; Stem Cells; Scaffolds

\section{How to Choose the Cells}

It seems best to choose autologous cells. But these cells are not easily available and are frequently in a pathological state. This explains the increasing interest in the use of undifferentiated progenitor cells which have ability to proliferate and differentiate into the target tissue. There are different types of stem cells (SC), which are characterised by their functional properties. After fertilisation, the egg divides into two, four and eight cells or identical blastomers. At the 16-cell stage, the embryo is made up of two external and internal cell types. Throughout the development of the embryo, then of the fotus and finally in the adult, different stem cells with different potential can be isolated and characterised: totipotent SC: up to the 8-blastomer stage. These cells are capable of inducing embryonic and fœtal development; pluripotent SC isolated from the morula, 16-32 cells and pluripotent SC isolated from the blastocyst. Embryonic cells are totipotent and theoretically have an unlimited life span. However, there is some controversy about using these cells due to their origin and procurement. Furthermore, they are affected by potential immunogenicity and tumorigenic transformation. In the fœtus, some stem cells circulate and can be isolated from the umbilical cord.

A particular case is that of the mesenchymal stem cells (MSC) isolated from different tissues (bone marrow, adipose tissue, umbilical cord, (Wharton Jelly) whose properties are similar to that of pluripotency $[1,2,5,10]$. Their physiological roles are concerned by their regenerative ability of injured tissue. They have been the subject of increasing attention in regenerative medicine thanks to their ability to differentiate into numerous cells lineages. Today, bone marrow is the most frequently used source of MSC, but it has been demonstrated that the number of
MSC decrease with the donor's age. For this reason, researchers identified alternative sources of extra-embryonic cells (foetal cord, amniotic membrane, placenta, etc.). To create distinct types of tissue from stem cells, control of the induction and maintenance of cell differentiation is indispensable.

\section{Scaffolds}

Tissue engineering scaffolds are designed to provide a threedimensional environment to support and direct cellular processes in their migration, proliferation, and differentiation toward a functional tissue. The scaffold provides mechanical stability and the required surface chemistry to orchestrate biological signals and influence cellular adhesion and function. Scaffolds must be biocompatible; sufficiently porous to allow nutrients to reach cells and ultimately degrade during biotissue development without toxic effects (see Table 1 below).

Table 1. Examples of available scaffolds for tissue Engineering (non exhaustive) (*scaffolds used by the authors in their researches).

\begin{tabular}{ll}
\hline Natural Polymers & Synthetic Polymers \\
\hline Hyaluronic Acid (HA)* & Poly ( $\alpha$-hydroxyl esters) (PLA, \\
Agarose* & PGA and PLGA)* \\
Sodium Alginate* & Poly (ethylen glycol/oxide ) \\
Cellulose & (PEG) \\
Chitosan & Poly - - lysine (PLL)* \\
Chondroitin sulfate & Poly (propylen fumarate) \\
Type I Collagen* & Poly (urethane) \\
Gelatin* & Poly (vinyl alcohol) \\
Fibrin & \\
\hline
\end{tabular}


Various ways have been explored for the development of 3-D scaffolds that will interact with cells. Biological scaffolds include collagen, alginate, hyaluronic acid, and polyelectrolyte films $[8,9,13]$. These scaffolds could allow better regulation of cell adhesion and matrix production by resident cells. However, biological scaffolds have a higher risk of contamination or immune reaction than synthetic scaffolds. On the other hand, synthetic materials, which are created de novo from molecules such as glycolic acid or lactic acid polymers, allow more precise control of both structural and mechanical properties, and of rates of resorption. Different kinds of hydrogels have been used for biomedical applications. Their water-swelling structure enables the time-dependent release or incorporation of water molecules in the outer environment. Alginates and polymers are widely employed and are very easy to use in cartilage engineering. However, few investigations have considered the organization of a tissue as a basis for the development of stratified tissues (e.g., cartilage, blood vessels). From this viewpoint, complex 3-D structures of a biotissue could a priori be built layer by layer using different components $[9,12]$. However, up to now, few methods that can efficiently synthesize stratified structures, including viable specific cells interacting with a biofunctionalized environment have been proposed. One example, we recently developed, is the construction of a stratified scaffold seeded with chondrocytes or SC by spraying. The stratified scaffold was constructed of layers of alginate / HA welded by polycation / polyanion (Poly-L-Lysine / HA) [12]. This new method allows the use of a 3D structure, which is important for chondrogenic induction of MSC with no growth factor, but also the construction of a stratified scaffold. The result is an innovative configuration that mimics the structure of cartilage. We clearly demonstrated that the sprayed biomaterial not only keeps the mechanical properties needed for cells, but also maintains the cell phenotype and the capacity to induce biocartilage. This original strategy could be used to design new stratified engineered tissues through progressive cells and hydrogel, with alternate layers sprayed with polyelectrolyte to weld the layers together. The spraying process helps form a highly functional and structured biotissues.

\section{Influence of Mechanical Forces}

All cells and tissues are subject to mechanical stresses [7]. These forces have various origins, from pressure forces linked to gravity, to motion forces (i.e., blood circulation, loading on cartilage and bone during movement). Their force ranges from a few Pascals on the vascular wall (shear stress) to several mega-Pascals on hip cartilage. It is now accepted that these forces are likely to modify cellular behaviour by affecting differentiation, metabolism, matrix production, paracrine or autocrine factor secretions, gene expression, etc. (concepts of mechanobiology and mechanotransduction). Such is the case for chondrocytes, bone cells, smooth muscle cells and vascular endothelial cells. However, although the physiological effects of stress are now well known, the steps leading from the mechanical signal to physiological responses and gene expression remain to be elucidated. Various hypotheses have been proposed but none is consistent with the bulk of experimental data. Most attempts to locate potential mechanotransducers have focused on two main structures of the cell: the membrane and the cytoskeleton, because forces act directly on the membrane and because the force applied is transmitted to the cytoskeleton. But it is likely that a cell uses a variety of mechanotransduction pathways depending on the type of physiological change induced, which makes interpretation difficult. In "biotissue" production, dynamic mechanical stresses on cells generally promote differentiation and increased matrix production.

\section{Cell Preparation and Engineering Approaches}

After choosing the cells, multiplication and differentiation in culture and/or in the scaffold are required. Thus, it is necessary to dispose of culture media whose composition, specific growth factors, etc., are compatible with the cells of interest and with a GMP process. A variety of proteins can affect cell differentiation and phenotypic expression, including transforming growth factor TGF- $\beta$, insulin-like growth factor (IGF-1), bone morphogenetic proteins (BMPs), fibroblast growth factors (FGFs), VEGF and epidermal growth factor (EGF). These molecules have a broad range of activities, including proliferation induction, differentiation and increasing the synthesis of the extracellular matrix components by the cells. For example transforming the growth factor (TGF- $\beta$ ) superfamily, including TGF- $\beta$ s 1-3 and insulin-like growth factor-1 (IGF-1), stimulate GAG synthesis by chondrocyte when collagen II is strongly stimulated by IGF-1. It has also been demonstrated that MSC cultures exposed to TGF- $\beta$ s show increased cell density, cluster formation, glycosaminoglycan and type II collagen synthesis.

\section{Grafting and Safety Studies: Respect of National Regulations}

One other problem to be solved is the local implantation (with or without scaffold). It is important to note that clinical applications of biotherapies are strictly controlled in Western countries. Cells or tissues of human origin can only be harvested in health centres accredited by Public Authorities (In France, different laws concern the authorization procedure related to the preparation, storage and clinical use of cells and tissues). The European Regulating Authorities are also very strict about the nature of the clinical trials and the choice of the patient.

Before grafting, different points need to be clarifed:

What type of grafting is planned for the patients? (What is the degree of severity of the pathology to consider, grafting in the early phase of the pathology or after stabilization? (i.e., myocardial infarction)

- Has the grafting site been identified or not?

- What are the benefits for the patient?

Clinical evaluation: this is a crucial step which must validate the graft through studies of total or partial tissue restoration.

- Is immunosuppression needed and what are the possible side effects

\section{Main Potential Clinical Applocations}

Biotissus based on stem cells have many possible applications in biology, in pharmacology, and hopefully in tissue engineering. The hopes concern the majority of the medical specialities, 
but different applications can be envisaged in the coming decade. In presentation different applications are presented (cartilage, bone, blood vessel, heart, liver, bladder reparation, etc.).

\section{Conclusions}

The regeneration of tissues is and will remain a challenge for the further development of cell therapy and tissue engineering. Adult stem cells and in particular mesenchymal SC should enable exciting developments in regenerative medicine. However, many problems remain to be solved:

- The lack of scientific and technical knowledge which could enable the development of innovative strategies (quality of the cells and their role in producing tissue repair, properties of the synthesized matrix, importance of mechanical signal, etc.)

- How can we increase the yield resulting from the preparation of the differentiated stem cells and advance our knowledge of the heterogeneous character of the preparations.

- to the need for a standardised reproducible product. The use of mesenchymal SC of Wharton's jelly seems to be an interesting possibility to prepare controlled batches of stem cells for the future.

- Technical problems regarding the definition of scaffolds, the cells used and long term stability and culture medium. In particular, the impact of the biomaterial used remains to be identified.

- Grafting: biotissue can be introduced via direct cell implantation (cell therapy), biotissue transplantation or gene therapy.

- Risk of teratogenic effects and of immune reaction (the immune risk is lowest using stem cells from the umbilical cord).

- Religious and legal issues concerning the different national regulations.

Current knowledge allows us to be optimistic about the future of tissue engineering and cell therapy but final answers can only be given after long-term randomised and controlled clinical studies.

\section{REFERENCES}

[1] Abdallah BM, Kassem M. Human mesenchymal stem cells: from basic biology to clinical applications. Gene Ther. 2008;

\section{5:109-116.}

[2] Barry F, Boynton RE, Liu B, Murphy JM. Chondrogenic differentiation of mesenchymal stem cells from bone marrow: differentiation-dependent gene expression of matrix components. Exp Cell Res. 2001;268:189-200.

[3] Berthelemy N, Kedjoudj H, Gaucher C, Schaaf P, Stoltz JF, Lacolley P, Voegel JC, Menu P. Polyelectrolyte films boost Progenitor Cell Differentiation into Endothelium-like Monolayers. Ad Mater. 2008;20:2674-2678.

[4] Cucchiarini M, Madry H. Gene therapy for cartilage defects. Gene Med. 2005;7:1495-1509.

[5] De Isla N, Huselstein C, Jessel N, Stoltz JF. Introduction to tissue engineering and application for cartilage engineering. Bio Medical Material and Engineering. 2010;20:127-133.

[6] Fisher JP, Mikos AG, Bronzino JD. Tissue Engineering. CRC Press (Boca Raton). 2007 (33 chapters).

[7] Fung YC. Biomechanics: Mechanical Properties of living Tissues. Springer-Verlag, New-York. 1981; 433 pp.

[8] Gigant-Huselstein C, Hubert P, Dumas D, Dellacherie E, Netter P, Payan E, Stoltz JF. Expression of adhesion molecules and collagen on rat chondrocyte seeded into alginate and hyaluronate based 3D bio systems. Influence of mechanical stresses. Biorheology. 2004;41:423-432.

[9] Kerdjoudj H, Berthelemy N, Rinckenbach S, Keardney-Schwartz A, Montagne K, Schaaf P, Lacolley P, Voegel JC, Menu P. Small Vessel Replacement by Human Umbilical Arteries With Polyelectrolyte Film-Treated Arteries. In Vivo Behavior. J Am Coll Cardiol. 2008;52:1589-1597.

[10] Stoltz JF. Mechanobiology: cartilage and chondrocyte. IOS Press (Amsterdam, Berlin, Tokyo). Biomedical and Health Research Series. 2000; Vol.1 - 190 pp; 2002; Vol. 2 - 288 pp; 2004; Vol. 3 - 446 pp; 2006; Vol. 4 - 443 pp, 2008 ; Vol. 5 - 357 pp.

[11] Stoltz JF, Bensoussan D, Decot V, Ciree A, Netter P, Gillet P. Cell and tissue engineering and clinical applications: an overview. Biomedical Material and Engineering. 2006;16:3-18.

[12] Tritz J, Rahouadj R, De Isla N, Charif N, Pinzano A, Mainard D, Bensoussan D, Netter P, Stoltz JF, Benkirane-Jessel N, Huselstein C. Designing a three-dimensional alginate hydrogel by spraying method for cartilage tissue engineering. Soft Matter. 2010;6:5165-5174.

[13] Wang Y, De Isla N, Decot D, Marchal G, Cauchois G, Huselstein C, Wang BH, Muller S, Netter P, Stoltz JF. Influences of construct properties on the proliferation and matrix synthesis of the differentiated chondrocytes cultured in alginate gel. Biorheology. 2008;45:527-238. 